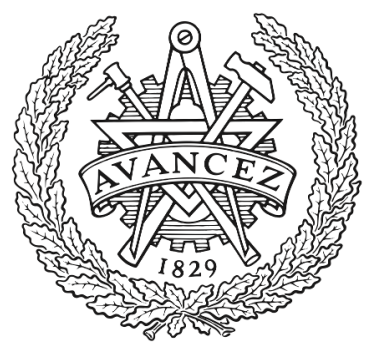

CHALMERS

UNIVERSITY OF TECHNOLOGY

\title{
Influence of Oxygen Vacancies on the Structure of $\mathrm{BiVO}<\mathrm{inf}>4</$ inf $>$
}

Downloaded from: https://research.chalmers.se, 2023-04-26 12:43 UTC

Citation for the original published paper (version of record):

Österbacka, N., Wiktor, J. (2021). Influence of Oxygen Vacancies on the Structure of

BiVO $<\inf >4</ \inf >$. Journal of Physical Chemistry C, 125(2): 1200-1207.

http://dx.doi.org/10.1021/acs.jpcc.0c08751

N.B. When citing this work, cite the original published paper. 


\title{
Influence of Oxygen Vacancies on the Structure of $\mathrm{BiVO}_{4}$
}

\author{
Nicklas Österbacka and Julia Wiktor*
}

Cite This: J. Phys. Chem. C 2021, 125, 1200-1207

Read Online

ABSTRACT: We study oxygen vacancies in the tetragonal scheelite phase of bismuth vanadate and identify stable oxygen-deficient structures. Upon subjecting these to variable-cell optimization, we find that oxygen vacancies give rise to significant structural distortions, the degree of which exhibits a vacancy concentration dependence. Furthermore, we show that these distortions give rise to splitting of powder X-ray diffraction peaks, yielding patterns similar to that of the monoclinic scheelite phase, and that these effects are also present at finite temperatures. Our results highlight the need for characterization methods beyond X-ray diffraction for identifying the phase of synthesized bismuth vanadate samples and the importance of oxygen partial pressure control during synthesis.

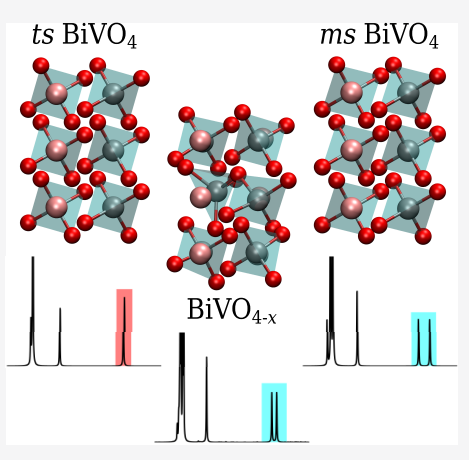

\section{INTRODUCTION}

Bismuth vanadate, $\mathrm{BiVO}_{4}$, is a promising candidate material for photoelectrochemical (PEC) water splitting due to the moderate band gap of around $2.4 \mathrm{eV}$ and favorable band alignment of its monoclinic scheelite phase with the water oxidation potential. ${ }^{1} \mathrm{BiVO}_{4}$ has been proposed as a model material providing a framework for studying phenomena underlying the PEC behavior of complex transition-metal oxides. ${ }^{2}$ For instance, it has been used as a development platform for new methods of increasing performance and stability of photocatalysts, such as photocharging ${ }^{3,4}$ and deposition of various cocatalysts or films on its surface. ${ }^{5-9}$

$\mathrm{BiVO}_{4}$ has been synthesized in three different phases: tetragonal zircon $\left(I 4_{1} /\right.$ amd: $a=b=7.310 \AA, c=6.462 \AA, \alpha=\beta$ $\left.=\gamma=90^{\circ}\right),{ }^{10}$ tetragonal scheelite $\left(14_{1} / a: a=b=5.147 \AA, c=\right.$ $\left.11.722 \AA, \alpha=\beta=\gamma=90^{\circ}\right),{ }^{11}$ and monoclinic scheelite $(I 2 / b: a$ $=5.194 \AA, b=5.090 \AA, c=11.697 \AA, \alpha=\beta=90^{\circ}, \gamma=$ $\left.90.387^{\circ}\right) .{ }^{11}$ An illustration of angle definitions is shown in Figure S1. The monoclinic scheelite $(\mathrm{ms})$ phase undergoes a reversible transition to tetragonal scheelite $(t s)$ at $528 \mathrm{~K}$, ${ }^{12}$ and an irreversible transition from tetragonal zircon to $m s$ occurs at $670-770 \mathrm{~K}^{13}$

The $m s$ structure of $\mathrm{BiVO}_{4}$ is the most stable phase at room temperature and has shown higher PEC efficiency than other synthesized forms. ${ }^{13,14}$ This has lead to a wealth of publications focused on studying and modifying $m s \mathrm{BiVO}_{4}$ photoanodes, but the performance of samples may vary greatly with crystallinity $^{15,16}$ and morphology ${ }^{17,18}$ and thus with the chosen synthesis method.

The choice of synthesis method, through variables such as temperature and atmosphere, is also tied to the preferential formation of defects within $\mathrm{BiVO}_{4}$ samples. ${ }^{19}$ The oxygen partial pressure during annealing is especially important, as it influences the concentration of oxygen defects. These will have a significant effect on many properties of the material. For instance, heat treatment in an oxygen-deficient atmosphere favors the formation of oxygen vacancies, ${ }^{20}$ which introduce charge transition levels within the band gap and can interact with excess charges in the lattice. ${ }^{21}$ The choice of synthesis precursor can also influence the final product. In stoichiometric $\mathrm{BiVO}_{4}$, vanadium has the $5+$ oxidation state. The use of precursors containing $\mathrm{V}^{4+}$, such as $\mathrm{VO}(\mathrm{acac})_{2}$, can lead to incomplete oxidation of $\mathrm{V}^{4+}$ to $\mathrm{V}^{5+}$ in the final product. This in turn results in an increase in the amount of oxygen vacancies in $\mathrm{BiVO}_{4}$ samples to maintain charge neutrality. ${ }^{22,23}$ The incomplete oxidation of vanadium can be unintentional, but it is also a common strategy for improving the efficiency of photoanodes based on $\mathrm{BiVO}_{4}{ }^{20,24-2720,24-27}$ However, the role of oxygen vacancies in $\mathrm{BiVO}_{4}$ is not entirely without controversy. It has been reported both that they enhance ${ }^{28}$ and diminish $^{29}$ the charge transfer properties of the material.

First-principles modeling through density functional theory (DFT) is commonly used to study the role of, e.g., defects ${ }^{30,31}$ and doping ${ }^{32}$ on the physical properties of metal oxides, but the predictive power of DFT is invariably tied to the exchange-correlation (XC) functional used. Using standard semilocal XC functionals is insufficient for systems where charge localization occurs since they preferentially delocalize charges due to the self-interaction error. $^{33,34}$ Hybrid XC functionals, where a fraction of the approximate exchange is replaced with the exact exchange formulation of the Hartree-

Received: September 25, 2020

Revised: December 16, 2020

Published: January 7, 2021 


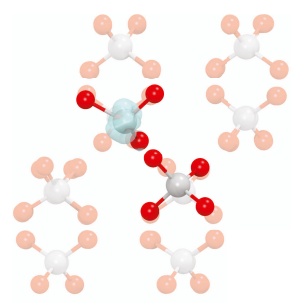

(a) Unbr.

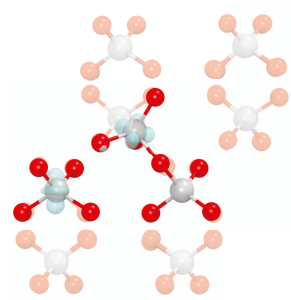

(e) $\mathrm{V}_{v} / \mathrm{V}_{v}^{c}$

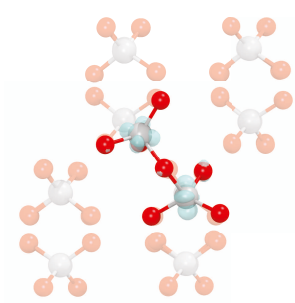

(b) $\mathrm{V}_{v} / \mathrm{V}_{b}$

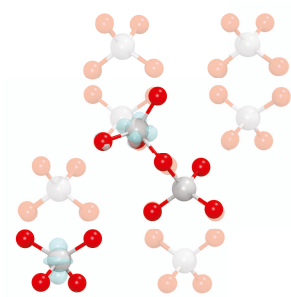

(f) $\mathrm{V}_{v} / \mathrm{V}_{v}^{f}$

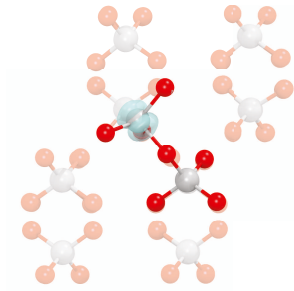

(c) $\mathrm{V}_{v} / \mathrm{V}_{v}$

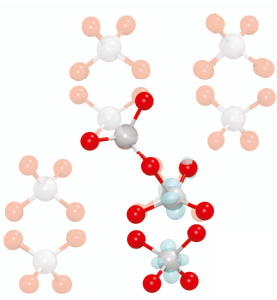

(g) $\mathrm{V}_{b} / \mathrm{V}_{b}^{c}$

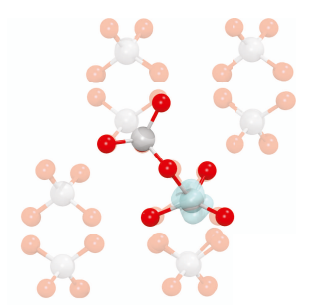

(d) $V_{b} / V_{b}$

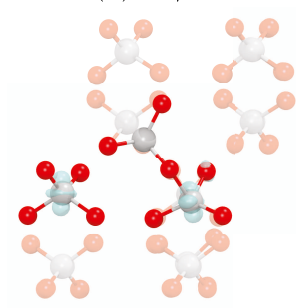

(h) $\mathrm{V}_{b} / \mathrm{V}_{h}^{f}$

Figure 1. Configurations containing one oxygen vacancy in the $2 \times 2 \times 1$ supercell. Isosurfaces represent electronic densities of the two excess electrons and are shown at $7.5 \%$ of their maximum values. $\mathrm{Bi}$ atoms and some $\mathrm{VO}_{4}$ units have been omitted for clarity.

Fock theory, correct this error allowing for a proper description of localized states. ${ }^{35}$ The choice of the exchange-correlation functional can also affect the calculated structural properties of a material. For the specific case of $\mathrm{BiVO}_{4}$, the Perdew-BurkeErnzerhof functional ${ }^{36}$ predicts that the $t$ phase is lower in energy, i.e., more stable, than the $m s$ phase, while a hybrid functional with an unphysically high fraction of exact exchange corrects this prediction. ${ }^{37}$

Numerous first-principles studies have been conducted on the effects of point defects ${ }^{21,38-41}$ and doping ${ }^{42-45}$ on the electronic structure and charge transfer properties of $\mathrm{BiVO}_{4}$. On the other hand, studies on their effects on the crystal structure of the material are scarce. Laraib et al. found that excess electrons can stabilize the $m s$ phase. ${ }^{46}$ However, they only considered delocalized electrons, while it has been shown that excess charges tend to localize in $\mathrm{BiVO}_{4} \cdot{ }^{47-50}$ Zhang et al. found that the presence of oxygen vacancies in $m s \mathrm{BiVO}_{4}$ leads to lattice contraction, ${ }^{51}$ but their calculations were only performed on the semilocal level of theory, thus not allowing for the full description of charge localization at the defects.

In this article, we attempt to bridge this knowledge gap by investigating the structural effects of oxygen vacancies at different concentrations, corresponding to supercells of different sizes, with varying local geometry in $\mathrm{BiVO}_{4}$ by means of hybrid DFT. We emphasize structural similarities to the stable $m s$ phase and show that the oxygen-deficient structures exhibit XRD patterns similar to that of pristine $m s$ $\mathrm{BiVO}_{4}$ upon cell relaxation.

\section{COMPUTATIONAL DETAILS}

All calculations were carried out using the Gaussian plane-wave method, as implemented in the CP2K package. ${ }^{52}$ Double- $\zeta$ polarized MOLOPT basis sets ${ }^{53}$ were used along with an auxiliary plane-wave basis set defined up to a cutoff energy of 600 Ry. Goedecker-Teter-Hutter pseudopotentials ${ }^{54}$ are used to describe core electrons. The PBE0-TC-LRC ${ }^{55,56}$ exchange-correlation functional with a 0.22 fraction of exact exchange was employed, and the auxiliary density matrix method $^{57}$ was used to speed up calculations. The Brillouin zone was sampled at the $\Gamma$ point.
We consider only triplet spin configurations in calculations. Seo et al. pointed out that the total energy difference between singlet and triplet configurations for $\mathrm{BiVO}_{4}$ with electrons localized at a neutral oxygen vacancy is only about $10 \mathrm{meV}^{21}$ We carried out a test calculation on the ts cell $2 \times 2 \times 1 \mathrm{~V}_{\mathrm{b}} / \mathrm{V}_{\mathrm{b}}^{\mathrm{c}}$ structure and confirmed that the energy difference is negligible, with the triplet roughly $1 \mathrm{meV}$ lower in energy.

The 95-atom supercell used in the present study is found by optimizing a $2 \times 2 \times 1$ repetition of a tetragonal $\mathrm{BiVO}_{4} I 2 / b$ structure while keeping its symmetry intact. The resulting conventional cell has lattice parameters $a=b=5.140 \AA$ and $c=$ $11.747 \AA$, in good agreement with the experimental values. The $3 \times 3 \times 1$ supercell is constructed using the same lattice parameters as the smaller one.

\section{RESULTS AND DISCUSSION}

Structure Optimization. We begin the analysis by investigating which oxygen vacancy-induced geometry distortions are stable in a $2 \times 2 \times 1$ repetition of an ideal ts lattice, containing 95 atoms in total. This corresponds to $\mathrm{BiVO}_{3.9375}$. As demonstrated by Seo et al., ${ }^{21}$ the oxygen vacancy in bismuth vanadate exists in numerous configurations, differing by the coordination of $\mathrm{V}$ atoms around the defect and by where the two excess electrons are localized. The first configuration, with an undercoordinated $\mathrm{VO}_{3}$, is formed by simply removing an oxygen atom and allowing atomic positions to relax, causing the two electrons to localize onto the $\mathrm{VO}_{3}$ while attracting a neighboring $\mathrm{VO}_{4}$. This unbridged configuration is shown in Figure $1 \mathrm{a}$. By bringing the neighboring $\mathrm{VO}_{4}$ and the $\mathrm{VO}_{3}$ units closer, we find that a bridged structure forms with one electron localizing onto each of the two $\mathrm{V}$ atoms, as shown in Figure $1 \mathrm{~b}$. Other configurations, shown in Figure $1 \mathrm{c}-\mathrm{h}$, are generated by inducing charge localization on different pairs of $\mathrm{V}$ atoms. This is done by elongating the $\mathrm{V}-\mathrm{O}$ bonds around select $\mathrm{V}$ atoms using the bridged structure as a template.

We remark that the two bridging $\mathrm{V}$ atoms in Figure $1 \mathrm{~b}$ are inequivalent, with the $\mathrm{V}$ atom of the vacancy displaced further from its pristine lattice position than the other bridging atom. This is shown clearly in Figure S2. These V atoms are labeled $V_{v}$, i.e., the $V$ with a vacancy, and $V_{b}$, i.e., the bridge-forming 
$\mathrm{VO}_{4}$. The $\mathrm{V}$ units furthest and closest to $\mathrm{V}_{\mathrm{v}}$ are denoted $\mathrm{V}_{\mathrm{v}}^{\mathrm{f}}$ and $\mathrm{V}_{\mathrm{v}}^{\mathrm{c}}$, with analogous definitions for $\mathrm{V}_{\mathrm{b}}^{\mathrm{f}}$ and $\mathrm{V}_{\mathrm{b}}^{\mathrm{c}}$. For brevity, we introduce a shorthand for the structures shown in Figure 1; for instance, the configuration shown in Figure $1 \mathrm{~b}$ is labeled $V_{\mathrm{v}} /$ $V_{b}$, i.e., electron localization onto both $V_{v}$ and $V_{b}$.

The energies relative to the unbridged configuration are shown in Figure 2. The lowest-energy configuration, $\mathrm{V}_{\mathrm{b}} / \mathrm{V}_{\mathrm{b}}^{\mathrm{c}}$, is

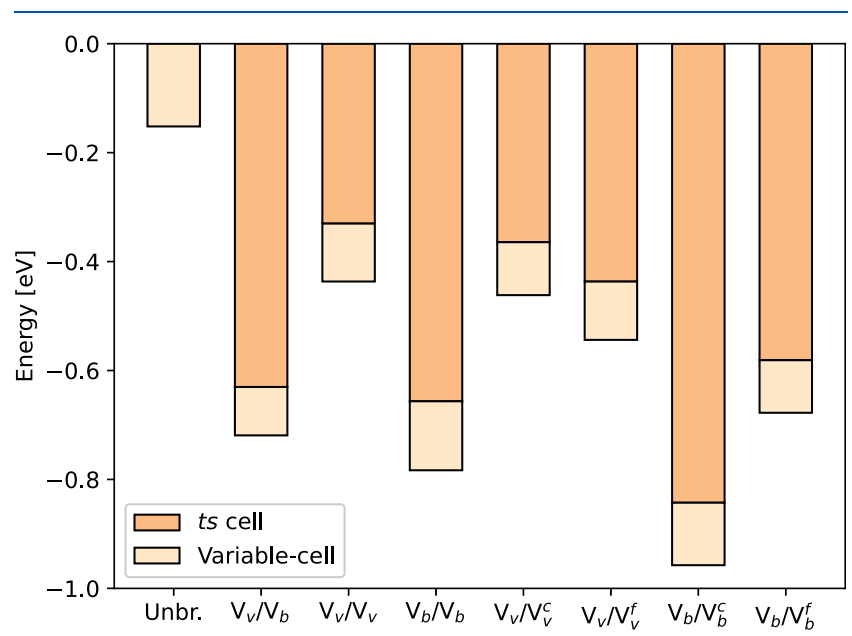

Figure 2. Total energies of various configurations of the oxygen vacancy in the neutral charge state in the $2 \times 2 \times 1$ supercell. All energies are given in reference to the unbridged configuration in the tetragonal cell.

about $186 \mathrm{meV}$ lower in energy than the next-lowest configuration, $\mathrm{V}_{\mathrm{b}} / \mathrm{V}_{\mathrm{b}}$. The other structures with electron localization into $V_{b}$ are closer in energy, with the greatest difference being about $75 \mathrm{meV}$ between $V_{b} / V_{b}$ and $V_{b} / V_{b}^{f}$. Localization onto $V_{b}$ is favored over localization onto $V_{v}$. Generally, we find that our results agree within $0.1 \mathrm{eV}$ with previous calculations on oxygen vacancies by Seo et al. ${ }^{21}$ The localization of electrons also introduces two states close to the middle of the band gap, in agreement with what Hegner et al. found for the bulk. ${ }^{41}$

We now investigate how the found relaxed oxygen vacancy configurations influence the crystal structure of bismuth vanadate. This is done by subjecting the previously considered structures to variable-cell optimization, i.e., allowing both atomic positions and the cell vectors of the simulation cell to relax. Energies of the configurations after cell optimization relative to the unbridged configuration in Figure 1a are shown in Figure 2. The deviation of the optimized cell from the ts lattice, and similarities to the $m s$ lattice, can be assessed based on how much the cell vector angles deviate from $90^{\circ}$ and on the length of the cell vectors, i.e., the lattice parameters.

The angle deviations from $90^{\circ}$ are shown in Figure 3. The lattice parameters are given in Table 1 and compared with the corresponding values for the defect-free optimized $2 \times 2 \times 1$ supercell as well as an experimental $m s$ structure from ref 11 . We remark that the deformations of the defective structures resemble the distortions of the monoclinic phase of $\mathrm{BiVO}_{4}$.

The relaxation of the cell results in a total energy reduction of about 100-150 meV for all geometries. The largest shift occurs for the unbridged configuration, but its energy remains the highest among all configurations considered here. Cell optimization does not affect the relative energies between structures. In other words, $\mathrm{V}_{\mathrm{b}} / \mathrm{V}_{\mathrm{b}}^{\mathrm{c}}$ remains the lowest-energy configuration. The relaxation also results in lattice expansion, with the degree of expansion varying between structures. The $a / b$ ratios of all structures differ from unity, with that of the unbridged configuration exhibiting a ratio very close to that of $m s \mathrm{BiVO}_{4}$. Cell angles now deviate from $90^{\circ}$, with a magnitude varying between structures. All configurations exhibit their strongest tilt in $\gamma$, and several of them lie close to the monoclinic angle of $\gamma=90.387^{\circ}$. This is coupled with a nonnegligible deviation in $\alpha$ and $\beta$ except for $\mathrm{V}_{\mathrm{v}} / \mathrm{V}_{\mathrm{v}}$, which differs significantly from the $m s$ angles only in $\beta$.

We have demonstrated that the introduction of oxygen vacancies in ts $\mathrm{BiVO}_{4}$ induces significant distortions in both atomic positions and the lattice itself. Upon removal of an oxygen atom, the two excess electrons localize, forming polarons. It is of interest to verify whether the observed distortions arise from the presence of vacancies, or if the polarons alone may give rise to this effect. To clarify this, we perform another calculation in which a triplet spin state is imposed to a pristine $2 \times 2 \times 1$ repetition of the conventional ts $\mathrm{BiVO}_{4}$ structure. This yields a neutral cell containing an electron-hole pair, similar to what would occur upon photoexcitation. To ensure polaronic localization of both charges, we additionally elongate the $\mathrm{V}-\mathrm{O}$ bond lengths in a $\mathrm{VO}_{4}$ unit. By monitoring the charge distribution, we verify that charge localization occurs in agreement with previous studies on polarons in $\mathrm{BiVO}_{4} \cdot{ }^{47,49,50}$ This system is then subjected to
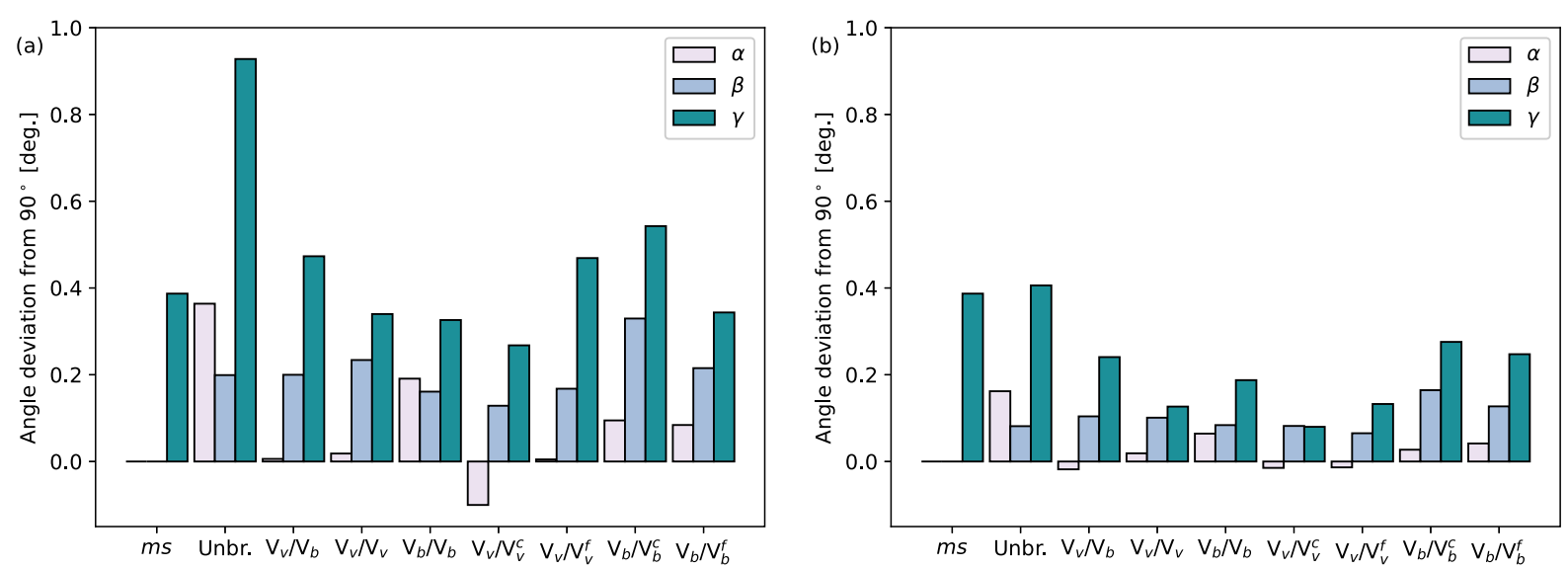

Figure 3. Cell vector angle deviations from $90^{\circ}$ of (a) $2 \times 2 \times 1\left(1.6 \%\right.$ vacancy ratio, $\left.\mathrm{BiVO}_{3.9375}\right)$ and (b) $3 \times 3 \times 1\left(0.7 \%, \mathrm{BiVO}_{3.972}\right)$ configurations. 
Table 1. Lattice Parameters for Optimized $2 \times 2 \times 1$ Supercell Configurations as well as Experimental $m s$ and Defect-Free Optimized $t$ S Structures

\begin{tabular}{|c|c|c|c|c|c|c|c|c|c|c|}
\hline & $m s$ & ts & unbr. & $\mathrm{V}_{\mathrm{v}} / \mathrm{V}_{\mathrm{b}}$ & $\mathrm{V}_{\mathrm{v}} / \mathrm{V}_{\mathrm{v}}$ & $\mathrm{V}_{\mathrm{b}} / \mathrm{V}_{\mathrm{b}}$ & $\mathrm{V}_{\mathrm{v}} / \mathrm{V}_{\mathrm{v}}^{\mathrm{c}}$ & $\mathrm{V}_{\mathrm{v}} / \mathrm{V}_{\mathrm{v}}^{\mathrm{f}}$ & $\mathrm{V}_{\mathrm{b}} / \mathrm{V}_{\mathrm{b}}^{\mathrm{c}}$ & $\mathrm{V}_{\mathrm{b}} / \mathrm{V}_{\mathrm{b}}^{\mathrm{f}}$ \\
\hline$a(\AA)$ & 5.194 & 5.140 & 5.222 & 5.184 & 5.187 & 5.180 & 5.172 & 5.189 & 5.187 & 5.176 \\
\hline$b(\AA)$ & 5.090 & 5.140 & 5.095 & 5.139 & 5.141 & 5.155 & 5.151 & 5.138 & 5.140 & 5.155 \\
\hline$c(\AA)$ & 11.697 & 11.747 & 11.794 & 11.766 & 11.783 & 11.784 & 11.777 & 11.780 & 11.780 & 11.752 \\
\hline
\end{tabular}

Table 2. Lattice Parameters of Oxygen-Deficient $3 \times 3 \times 1$ Configurations

\begin{tabular}{|c|c|c|c|c|c|c|c|c|}
\hline & unbr. & $\mathrm{V}_{\mathrm{v}} / \mathrm{V}_{\mathrm{b}}$ & $\mathrm{V}_{\mathrm{v}} / \mathrm{V}_{\mathrm{v}}$ & $\mathrm{V}_{\mathrm{b}} / \mathrm{V}_{\mathrm{b}}$ & $\mathrm{V}_{\mathrm{v}} / \mathrm{V}_{\mathrm{v}}^{\mathrm{c}}$ & $\mathrm{V}_{\mathrm{v}} / \mathrm{V}_{\mathrm{v}}^{\mathrm{f}}$ & $\mathrm{V}_{\mathrm{b}} / \mathrm{V}_{\mathrm{b}}^{\mathrm{c}}$ & $\mathrm{V}_{\mathrm{b}} / \mathrm{V}_{\mathrm{b}}^{\mathrm{f}}$ \\
\hline$a(\AA)$ & 5.175 & 5.160 & 5.159 & 5.159 & 5.153 & 5.156 & 5.163 & 5.162 \\
\hline$b(\AA)$ & 5.117 & 5.138 & 5.141 & 5.145 & 5.145 & 5.143 & 5.138 & 5.141 \\
\hline$c(\AA)$ & 11.767 & 11.750 & 11.758 & 11.761 & 11.754 & 11.753 & 11.752 & 11.749 \\
\hline
\end{tabular}

variable-cell optimization, yielding a lattice defined by $(a=$ $5.145 \AA, b=5.134 \AA, c=11.737 \AA, \alpha=90.052^{\circ}, \beta=90.030^{\circ}, \gamma$ $\left.=90.015^{\circ}\right)$. The lattice distortions are thus much smaller than in the presence of oxygen vacancies, and we conclude that polarons by themselves do not contribute significantly to the distortions observed for the oxygen-deficient structures.

The formation energy of oxygen vacancies in $\mathrm{BiVO}_{4}$ is predicted to be low, ${ }^{21,42}$ and their formation during synthesis is thus unavoidable, with a concentration depending on the conditions of the chosen synthesis procedure. The $2 \times 2 \times 1$ supercell considered above corresponds to a vacancy atomic ratio $\left(\frac{N_{\mathrm{vac}}}{N_{\mathrm{O}}}\right)$ of $1.6 \%$, and we now consider the effects of lowervacancy concentrations by repeating the optimization procedure with a $3 \times 3 \times 1$ supercell containing 216 atoms, corresponding to a ratio of $0.7 \%$, i.e., $\mathrm{BiVO}_{3.972}$. The energies relative to the unbridged configuration are shown in Figure S3, while the cell vector angles and lattice parameters are shown in Figure 3 and Table 2, respectively.

The energy reduction from cell relaxation is about 100-130 $\mathrm{meV}$, and localization onto $\mathrm{V}_{\mathrm{b}}$ is again favored over $\mathrm{V}_{\mathrm{v}}$, with energy differences smaller than in the 95-atom supercell. The largest shift occurs for the $\mathrm{V}_{\mathrm{b}} / \mathrm{V}_{\mathrm{b}}^{\mathrm{c}}$ configuration, which is also the most stable, though the differences in energy reduction are smaller than the previous values. We observe distortions similar to those of the smaller supercell, albeit with smaller cell angles. Our results here demonstrate that the vacancy-induced distortions exhibit a concentration dependence, with lower concentrations yielding smaller distortions, as expected.

Powder X-ray Diffraction Patterns (PXRD). Powder Xray diffraction (PXRD) is a method commonly used for materials characterization and has frequently been used to determine the phase of synthesized $\mathrm{BiVO}_{4}$ samples during the past few decades. ${ }^{11,14}$ We now investigate if the oxygen vacancy-induced distortions would be apparent in PXRD patterns by means of simulations using the software VESTA. ${ }^{58}$ The wavelength chosen, $1.5406 \AA$, corresponds to $\mathrm{Cu} \mathrm{K} \alpha$ radiation.

While the $\mathrm{Cu} \mathrm{K} \alpha$ PXRD patterns of $t$ and $m s \mathrm{BiVO}_{4}$ are similar, they both have some characteristic discrepancies that have been used for phase identification. ${ }^{14}$ The ts phase exhibits single reflections at $2 \theta$ values of about $18.5,35$, and $46^{\circ}$, corresponding to crystal planes with the Miller indices of $(1,0,1),(2,0,0)$, and $(2,0,4)$. The $m s$ phase exhibits splitting of these reflections corresponding to Miller indices of $[(1,0,1)$, $(0,1,1)],[(2,0,0),(0,2,0)]$ and $[(2,0,4),(0,2,4)]$ as well as an additional reflection at $2 \theta=15^{\circ}$ corresponding to the plane $(0,0,2)$. In addition, the $m s$ phase exhibits peak splitting at $\sim 50^{\circ}$ as well as the formation of peak clusters at $\sim 40$ and $\sim 60^{\circ}$ rather than clear individual peaks.

The simulated PXRD patterns of experimental ts and $m s$ structures are shown in Figure 4 in addition to those of the $V_{v}$ /

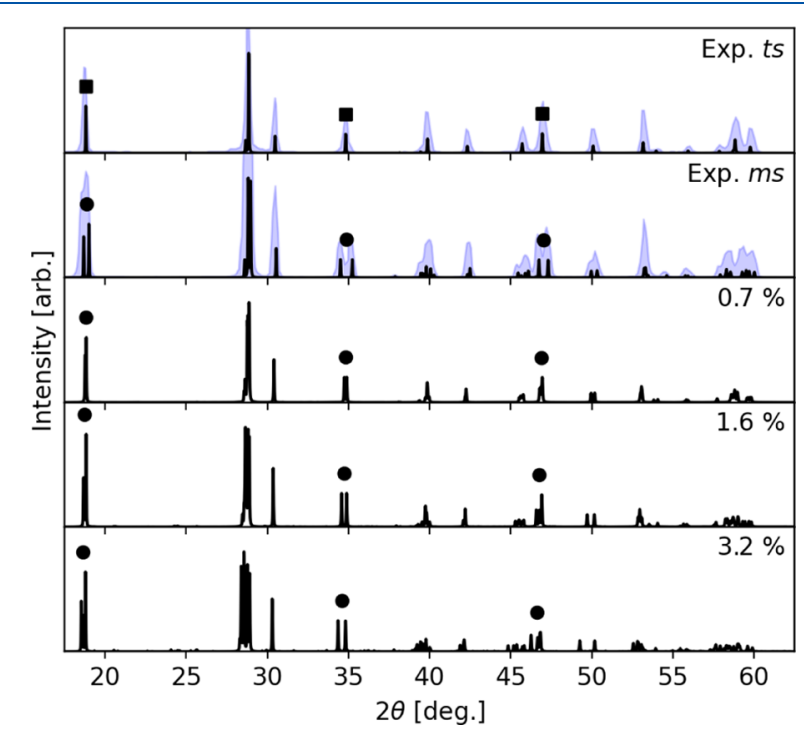

Figure 4. Simulated powder $\mathrm{XRD}$ patterns of oxygen-deficient $\mathrm{BiVO}_{4}$ $\mathrm{V}_{\mathrm{v}} / \mathrm{V}_{\mathrm{b}}$ structures (solid lines). Experimental ts and $m s$ patterns were extracted from ref 14 (blue shading). Squares and circles denote marker peaks used to identify $m s$ and ts phases, respectively, as per Tokunaga et al. The intensities of the experimental patterns have been exaggerated to highlight similarities and differences to the simulated patterns.

$\mathrm{V}_{\mathrm{b}}$ structures in the $2 \times 2 \times 1$ (oxygen vacancy atomic ratio of $1.6 \%)$ and $3 \times 3 \times 1(0.7 \%)$ supercells. Experimental patterns for pure ts and $\mathrm{ms} \mathrm{BiVO}_{4}$ from Tokunaga et al. ${ }^{14}$ are also shown. Since even higher concentrations of oxygen vacancies were observed experimentally close to the surface (as high as $15 \%),{ }^{24,59}$ we also assess the effect of increasing the defect ratio. This is done by placing two symmetrically equivalent vacancies in the $V_{v} / V_{b}$ configuration in the $2 \times 2 \times 1$ supercell, leading to an oxygen vacancy atomic ratio of $3.2 \%$, i.e., $\mathrm{BiVO}_{3.875}$.

The experimentally determined patterns agree well with the corresponding simulated patterns. However, experiments fail to capture some of the finer details for the ms pattern. The splitting of peaks at $28^{\circ}$ manifests as a single wide peak and the broadening of the peak cluster at $\sim 60^{\circ}$ makes it difficult to distinguish separate peaks, for instance. For all concentrations of oxygen vacancies, the peak splitting at $2 \theta$ values of $18.5,35$, 
46 , and $50^{\circ}$ is apparent in the optimized structures. They also exhibit the formation of peak clusters at $\sim 40$ and $\sim 60^{\circ}$. This indicates that oxygen-deficient $\mathrm{BiVO}_{4}$ samples should exhibit peak splitting similar to that of the pristine $m s$ structure. The degree of peak splitting at 35,46 , and $50^{\circ}$ increases with vacancy concentration, with $3.2 \%$ ratio being most pronounced of the optimized systems considered here.

It has been suggested by Kim and Lee that lesser splitting of the peak around $19^{\circ}$ may indicate a mixture of $t s$ and $m s$ phases. $^{60}$ The spectra of oxygen-deficient structures here exhibit a weaker splitting of that peak than the experimental $m s$ structure, demonstrating that this phenomenon may arise due to cell distortions induced by oxygen vacancies as well. Our results indicate that PXRD is insufficient to unambiguously determine whether the phase of synthesized $\mathrm{BiVO}_{4}$ samples is monoclinic scheelite unless a significant concentration of oxygen vacancies is ruled out. Moreover, the fact that oxygen vacancies induce such a pronounced structural modification, even at low concentrations, signifies the importance of oxygen potential control during material synthesis. Further studies are needed to assess the relationship between oxygen vacancy concentrations and synthesis conditions.

The PXRD patterns for all optimized structures are shown in Figures S4 and S5. They all exhibit the $m s$-like splitting of peaks, although the degree of splitting varies somewhat. The distance between the two peaks at $\sim 35^{\circ}$ increases with the $\gamma$ angle, for instance. Since they are otherwise similar to each other, only the $V_{v} / V_{b}$ structure is shown in Figure 4 as its polaron configuration fits well into the $3.2 \%$ structure.

Stability of Defects. Experimental studies on $\mathrm{BiVO}_{4}$ are typically done at room temperature. So far, our calculations have corresponded to a temperature of $0 \mathrm{~K}$. To verify that the vacancy-induced distortions studied here remain present at finite temperatures, we perform a 6 ps NPT ab initio molecular dynamics simulation with the lowest-energy configuration $\left(V_{b} /\right.$ $\mathrm{V}_{\mathrm{b}}^{\mathrm{c}}$ ) at $300 \mathrm{~K}$ and 1 bar with a 2 fs timestep. We discard the first 1.2 ps to account for equilibration effects.

Cell vector angles and lattice parameters from both the MD simulation and corresponding cell optimization are shown in Figure 5 and Table 3, respectively. While temperature does affect the cell parameters, the effect is rather marginal. Lattice parameters increase by about $0.02 \AA$ in $a$ and $b$ and by about

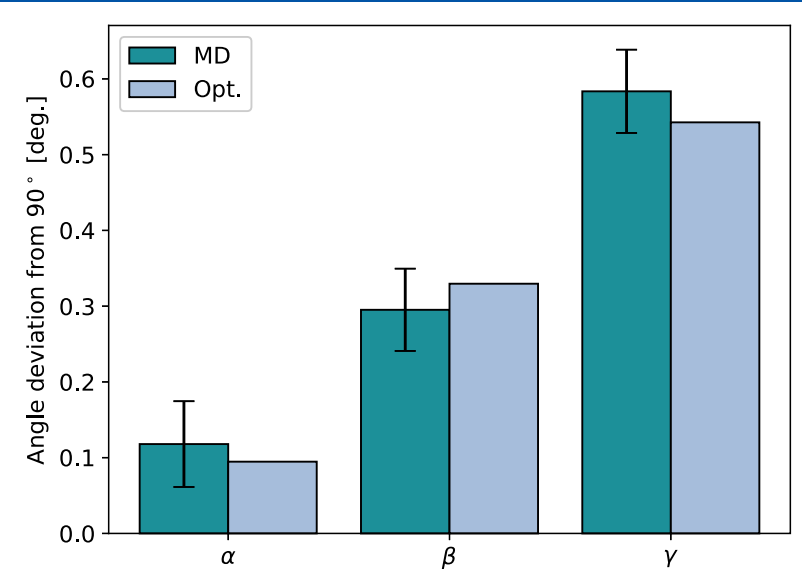

Figure 5. Average angle deviation from $90^{\circ}$ during 6 ps of molecular dynamics at $300 \mathrm{~K}$ and $1 \mathrm{bar}$ as well as for the starting configuration. The error bars are standard error of the mean estimates based on a blocking analysis.
Table 3. Lattice Parameters of the $V_{b} / V_{b}^{c}$ Configuration of Figure $\mathbf{1 g}^{a}$

\begin{tabular}{crc} 
& \multicolumn{1}{c}{ MD } & Opt. \\
$a(\AA)$ & $5.206 \pm 0.007$ & 5.187 \\
$b(\AA)$ & $5.159 \pm 0.006$ & 5.140 \\
$c(\AA)$ & $11.859 \pm 0.008$ & 11.780
\end{tabular}

${ }^{a}$ Uncertainties are standard error of the mean estimates based on a blocking analysis.

$0.08 \AA$ in $c$ on average, while cell vector angles remain the same within error margins. These results indicate that the oxygendeficient structures remain distorted at finite temperatures.

Comparison with Experiments. We now refer to the literature to investigate how experimental PXRD patterns compared to those of the oxygen-deficient samples presented in this work. The three experimental studies discussed below have all described the synthesis of $\mathrm{BiVO}_{4}$ and indexed their samples to the $m s$ phase, despite the lack of clear $m s$-like characteristics in PXRD patterns.

Luo et al. ${ }^{61}$ have presented a novel technique of $\mathrm{BiVO}_{4}$ nanoparticle synthesis involving ball-milling and subsequent annealing at different temperatures. The characteristic peak splitting at 19 and $35^{\circ}$ is evident in the samples annealed at $500{ }^{\circ} \mathrm{C}$ and above, but not in the samples treated at lower temperatures. The authors nevertheless index all annealed samples to the $m s$ and highlight the $400{ }^{\circ} \mathrm{C}$ sample as having a good balance between crystallization and particle size distribution. The PXRD pattern shows no peak splitting at $35^{\circ}$ and has a wide peak cluster at $60^{\circ}$.

Appavu et al. ${ }^{62}$ have synthesized a photocatalyst consisting of $\mathrm{BiVO}_{4}$ and nitrogen-reduced graphene oxide. As part of the study, they have also synthesized pure $\mathrm{BiVO}_{4}$. The PXRD pattern for the $\mathrm{BiVO}_{4}$ sample exhibits rather poor splitting of the $35^{\circ}$ peak, manifesting as a wider single peak than that of the pure ts phase. The pattern also exhibits a wide peak cluster at $60^{\circ}$ rather than individual peaks.

Zalfani et al. ${ }^{63}$ have prepared $\mathrm{BiVO}_{4} / \mathrm{TiO}_{2}$ nanocomposites and presented a PXRD pattern for the as-synthesized $\mathrm{BiVO}_{4}$. The pattern does not exhibit clear splitting of the 35 and $47^{\circ}$ peaks, although the peaks are wider and flatter than those of the ts pattern of Tokunaga et al. This suggests that the peaks are indeed split, but that the degree of splitting is less than would be expected from a phase-pure $m s$ sample. The pattern also exhibits a wide and flat peak at $60^{\circ}$ rather than the welldefined individual peaks characteristic of the $t$ phase.

It is clear that experimental PXRD patterns are not always unambiguous. In fact, the PXRD patterns of the experiments highlighted here are more similar to our oxygen-deficient PXRD patterns than those of the $m s$ phase of Tokunaga et al., suggesting that their synthesis methods may have resulted in oxygen-deficient samples instead. In particular, the patterns appear to show a lesser degree of peak splitting in line with what we observe for the defective structures, as well as a wide and flat peak around $60^{\circ}$. The peak cluster at $60^{\circ}$ in the oxygen-deficient structures of Figure 4 shows several distinct peaks, but as discussed earlier, experiments do not capture the same level of detail as simulations do.

Complementary Characterization Techniques. In cases where PXRD is insufficient for unambiguous phase assignment of synthesized $\mathrm{BiVO}_{4}$, such as the ones highlighted above, using complementary characterization techniques to probe samples for the presence of oxygen vacancies would be 
appropriate. There are several methods that may be used to this end. Yao et al. ${ }^{64}$ used energy-dispersive X-ray spectroscopy and found that their nanosheets are oxygen-deficient. Raman spectroscopy is another useful technique as it is highly sensitive to changes in local chemical environment. It has been suggested that oxygen vacancies induce a broadening and shift of Raman peaks in $\mathrm{BiVO}_{4}$ photoanodes. ${ }^{65} \mathrm{~A}$ similar effect has been observed for nanoparticles, but it was attributed to differences in sample size and shape. ${ }^{18}$

As previously mentioned, the formation of oxygen vacancies is coupled to the formation of $\mathrm{V}^{4+}$ species in $\mathrm{BiVO}_{4}$. This has successfully been used to probe synthesized samples for this type of defect. Selim et al. $^{23}$ used X-ray photoelectron spectroscopy to obtain an estimate of the concentration close to the surface by comparing the relative signals of $\mathrm{V}\left(2 \mathrm{p}_{3 / 2}\right)$ states from $\mathrm{V}^{5+}$ and $\mathrm{V}^{4+}$. The vacancy-related $\mathrm{V}^{4+}$ is paramagnetic, which Tan et al. ${ }^{20}$ used to investigate the relative concentrations of oxygen vacancies in $\mathrm{BiVO}_{4}$ samples annealed in argon at different temperatures through electron paramagnetic resonance spectroscopy. This technique, unlike the others discussed here, has the additional benefit of allowing for the detection of oxygen vacancies in the bulk.

\section{CONCLUSIONS}

In summary, we have studied the effect of oxygen vacancies on the structure of bismuth vanadate. Starting from a ts structure, we first investigated which oxygen-deficient geometries are stable. We then subjected them to variable-cell optimization, yielding significant distortions of the lattice. The distortions increase with oxygen vacancy concentration and cannot arise only due to the presence of polarons. Furthermore, we demonstrated that the distortions result in PXRD peak splittings similar to that commonly associated with the $m s$ structure and that the distortions are retained at room temperature. Finally, we discuss our results in relation to experimental PXRD studies and find striking similarities between our simulated patterns and those of experiments. Our results imply that PXRD alone is insufficient to assign the $m s$ phase to synthesize bismuth vanadate samples in cases where the patterns are ambiguous. One must also rule out the presence of oxygen vacancies at significant concentrations. They also highlight the importance of oxygen partial pressure control during synthesis and cast doubt on whether the synthesis methods found in the literature, supposedly resulting in samples of pure $m s \mathrm{BiVO}_{4}$, may in fact yield oxygen-deficient materials instead.

\section{ASSOCIATED CONTENT}

\section{SI Supporting Information}

The Supporting Information is available free of charge at https://pubs.acs.org/doi/10.1021/acs.jpcc.0c08751.

Definition of crystallographic angles, inequivalence of $\mathrm{V}_{\mathrm{v}}$ and $V_{b}$, stability of $3 \times 3 \times 1$ configurations, PXRD patterns for all optimized structures, and tests on the parametrization of the hybrid functional (PDF)

\section{AUTHOR INFORMATION}

\section{Corresponding Author}

Julia Wiktor - Department of Physics, Chalmers University of Technology, SE-41296 Gothenburg, Sweden; (1) orcid.org/ 0000-0003-3395-1104; Email: julia.wiktor@chalmers.se

\section{Author}

Nicklas Österbacka - Department of Physics, Chalmers

University of Technology, SE-412 96 Gothenburg, Sweden; (1) orcid.org/0000-0002-6043-4607

Complete contact information is available at:

https://pubs.acs.org/10.1021/acs.jpcc.0c08751

\section{Notes}

The authors declare no competing financial interest.

\section{ACKNOWLEDGMENTS}

The authors acknowledge funding from the "Area of Advance-Materials Science" at Chalmers University of Technology and the Swedish Research Council (201903993). The computations were performed on resources provided by the Swedish National Infrastructure for Computing (SNIC) at NSC and PDC.

\section{REFERENCES}

(1) Park, Y.; McDonald, K. J.; Choi, K.-S. Progress in Bismuth Vanadate Photoanodes for Use in Solar Water Oxidation. Chem. Soc. Rev. 2013, 42, 2321-2337.

(2) Sharp, I. D.; Cooper, J. K.; Toma, F. M.; Buonsanti, R. Bismuth Vanadate as a Platform for Accelerating Discovery and Development of Complex Transition-Metal Oxide Photoanodes. ACS Energy Lett. 2017, 2, 139-150.

(3) Trześniewski, B. J.; Smith, W. A. Photocharged $\mathrm{BiVO}_{4}$ Photoanodes for Improved Solar Water Splitting. J. Mater. Chem. A 2016, 4, 2919-2926.

(4) Liu, E. Y.; Thorne, J. E.; He, Y.; Wang, D. Understanding Photocharging Effects on Bismuth Vanadate. ACS Appl. Mater. Interfaces 2017, 9, 22083-22087.

(5) Kim, T. W.; Choi, K.-S. Nanoporous $\mathrm{BiVO}_{4}$ Photoanodes with Dual-Layer Oxygen Evolution Catalysts for Solar Water Splitting. Science 2014, 343, 990-994.

(6) McDowell, M. T.; Lichterman, M. F.; Spurgeon, J. M.; Hu, S.; Sharp, I. D.; Brunschwig, B. S.; Lewis, N. S. Improved Stability of Polycrystalline Bismuth Vanadate Photoanodes by Use of Dual-Layer Thin $\mathrm{TiO}_{2} / \mathrm{Ni}$ Coatings. J. Phys. Chem. C 2014, 118, 19618-19624.

(7) Li, R.; Han, H.; Zhang, F.; Wang, D.; Li, C. Highly Efficient Photocatalysts Constructed by Rational Assembly of Dual-Cocatalysts Separately on Different Facets of $\mathrm{BiVO}_{4}$. Energy Environ. Sci. 2014, 7, $1369-1376$

(8) Palaniselvam, T.; Shi, L.; Mettela, G.; Anjum, D. H.; Li, R.; Katuri, K. P.; Saikaly, P. E.; Wang, P. Vastly Enhanced $\mathrm{BiVO}_{4}$ Photocatalytic OER Performance by $\mathrm{NiCoO}_{2}$ as Cocatalyst. Adv. Mater. Interfaces 2017, 4, No. 1700540.

(9) Kim, T. W.; Choi, K.-S. Improving Stability and Photoelectrochemical Performance of $\mathrm{BiVO}_{4}$ Photoanodes in Basic Media by Adding a $\mathrm{ZnFe}_{2} \mathrm{O}_{4}$ Layer. J. Phys. Chem. Lett. 2016, 7, 447-451.

(10) Li, G.; Bai, Y.; Zhang, W. F. Difference in Valence Band Top of $\mathrm{BiVO}_{4}$ with Different Crystal Structure. Mater. Chem. Phys. 2012, 136, 930-934.

(11) Sleight, A. W.; Chen, H.-y.; Ferretti, A.; Cox, D. E. Crystal Growth and Structure of $\mathrm{BiVO}_{4}$. Mater. Res. Bull. 1979, 14, 15711581.

(12) Bierlein, J. D.; Sleight, A. W. Ferroelasticity in $\mathrm{BiVO}_{4}$. Solid State Commun. 1975, 16, 69-70.

(13) Kudo, A.; Omori, K.; Kato, H. A Novel Aqueous Process for Preparation of Crystal Form-Controlled and Highly Crystalline $\mathrm{BiVO}_{4}$ Powder from Layered Vanadates at Room Temperature and Its Photocatalytic and Photophysical Properties. J. Am. Chem. Soc. 1999, 121, 11459-11467.

(14) Tokunaga, S.; Kato, H.; Kudo, A. Selective Preparation of Monoclinic and Tetragonal $\mathrm{BiVO}_{4}$ with Scheelite Structure and Their Photocatalytic Properties. Chem. Mater. 2001, 13, 4624-4628. 
(15) Li, H.; Liu, G.; Duan, X. Monoclinic $\mathrm{BiVO}_{4}$ with Regular Morphologies: Hydrothermal Synthesis, Characterization and Photocatalytic Properties. Mater. Chem. Phys. 2009, 115, 9-13.

(16) Castillo, N. C.; Heel, A.; Graule, T.; Pulgarin, C. FlameAssisted Synthesis of Nanoscale, Amorphous and Crystalline, Spherical $\mathrm{BiVO}_{4}$ with Visible-Light Photocatalytic Activity. Appl. Catal., B 2010, 95, 335-347.

(17) Zhao, Y.; Li, R.; Mu, L.; Li, C. Significance of Crystal Morphology Controlling in Semiconductor-Based Photocatalysis: A Case Study on $\mathrm{BiVO}_{4}$ Photocatalyst. Cryst. Growth Des. 2017, 17, 2923-2928.

(18) Yu, J.; Kudo, A. Effects of Structural Variation on the Photocatalytic Performance of Hydrothermally Synthesized $\mathrm{BiVO}_{4}$. Adv. Funct. Mater. 2006, 16, 2163-2169.

(19) Lamers, M.; Fiechter, S.; Friedrich, D.; Abdi, F.; van de Krol, F. R. Formation and Suppression of Defects during Heat Treatment of $\mathrm{BiVO}_{4}$ Photoanodes for Solar Water Splitting. J. Mater. Chem. A 2018, 6, 18694-18700.

(20) Tan, H. L.; Suyanto, A.; Denko, A. T. D.; Saputera, W. H.; Amal, R.; Osterloh, F. E.; Ng, Y. H. Enhancing the Photoactivity of Faceted $\mathrm{BiVO}_{4}$ via Annealing in Oxygen-Deficient Condition. Part. Part. Syst. Charact. 2017, 34, No. 1600290.

(21) Seo, H.; Ping, Y.; Galli, G. Role of Point Defects in Enhancing the Conductivity of $\mathrm{BiVO}_{4}$. Chem. Mater. 2018, 30, 7793-7802.

(22) Byun, S.; Jung, G.; Shi, Y.; Lanza, M.; Shin, B. Aging of a Vanadium Precursor Solution: Influencing Material Properties and Photoelectrochemical Water Oxidation Performance of SolutionProcessed $\mathrm{BiVO}_{4}$ Photoanodes. Adv. Funct. Mater. 2020, 30, No. 1806662.

(23) Selim, S.; Pastor, E.; García-Tecedor, M.; Morris, M. R.; Francàs, L.; Sachs, M.; Moss, B.; Corby, S.; Mesa, C. A.; Gimenez, S.; et al. Impact of Oxygen Vacancy Occupancy on Charge Carrier Dynamics in $\mathrm{BiVO}_{4}$ Photoanodes. J. Am. Chem. Soc. 2019, 141, 18791-18798.

(24) Wang, S.; Chen, P.; Bai, Y.; Yun, J.-H.; Liu, G.; Wang, L. New $\mathrm{BiVO}_{4}$ Dual Photoanodes with Enriched Oxygen Vacancies for Efficient Solar-Driven Water Splitting. Adv. Mater. 2018, 30, No. 1800486

(25) Qin, D.-D.; Wang, T.; Song, Y.-M.; Tao, C.-L. Reduced Monoclinic $\mathrm{BiVO}_{4}$ for Improved Photoelectrochemical Oxidation of Water under Visible Light. Dalton Trans. 2014, 43, 7691-7694.

(26) Zhang, Y.; Guo, Y.; Duan, H.; Li, H.; Sun, C.; Liu, H. Facile Synthesis of $\mathrm{V}^{4+}$ Self-Doped, [010] Oriented $\mathrm{BiVO}_{4}$ Nanorods with Highly Efficient Visible Light-Induced Photocatalytic Activity. Phys. Chem. Chem. Phys. 2014, 16, 24519-24526.

(27) Wu, J.-M.; Chen, Y.; Pan, L.; Wang, P.; Cui, Y.; Kong, D.; Wang, L.; Zhang, X.; Zou, J.-J. Multi-Layer Monoclinic $\mathrm{BiVO}_{4}$ with Oxygen Vacancies and $\mathrm{V}^{4+}$ Species for Highly Efficient Visible-Light Photoelectrochemical Applications. Appl. Catal., B 2018, 221, 187195.

(28) Zhang, W.; Song, L.; Cen, J.; Liu, M. Mechanistic Insights into Defect-Assisted Carrier Transport in Bismuth Vanadate Photoanodes. J. Phys. Chem. C 2019, 123, 20730-20736.

(29) Qiu, W.; Xiao, S.; Ke, J.; Wang, Z.; Tang, S.; Zhang, K.; Qian, W.; Huang, Y.; Huang, D.; Tong, Y.; et al. Freeing the Polarons to Facilitate Charge Transport in $\mathrm{BiVO}_{4}$ from Oxygen Vacancies with an Oxidative 2D Precursor. Angew. Chem. 2019, 131, 19263-19271.

(30) Janotti, A.; Varley, J. B.; Rinke, P.; Umezawa, N.; Kresse, G.; Van de Walle, C. G. Hybrid Functional Studies of the Oxygen Vacancy in $\mathrm{TiO}_{2}$. Phys. Rev. B 2010, 81, No. 085212.

(31) Tian, F.; Zhao, L.; Xue, X.-Y.; Shen, Y.; Jia, X.; Chen, S.; Wang, Z. DFT Study of CO Sensing Mechanism on Hexagonal $\mathrm{WO}_{3}(001)$ Surface: The Role of Oxygen Vacancy. Appl. Surf. Sci. 2014, 311, 362-368.

(32) Chen, H.; Li, X.; Wan, R.; Kao-Walter, S.; Lei, Y.; Leng, C. A DFT Study on Modification Mechanism of $(\mathrm{N}, \mathrm{S})$ Interstitial CoDoped Rutile $\mathrm{TiO}_{2}$. Chem. Phys. Lett. 2018, 695, 8-18.
(33) Zhang, Y.; Yang, W. A Challenge for Density Functionals: SelfInteraction Error Increases for Systems with a Noninteger Number of Electrons. J. Chem. Phys. 1998, 109, 2604-2608.

(34) Pacchioni, G.; Frigoli, F.; Ricci, D.; Weil, J. A. Theoretical Description of Hole Localization in a Quartz Al Center: The Importance of Exact Electron Exchange. Phys. Rev. B 2000, 63, No. 054102.

(35) Miceli, G.; Chen, W.; Reshetnyak, I.; Pasquarello, A. Nonempirical Hybrid Functionals for Band Gaps and Polaronic Distortions in Solids. Phys. Rev. B 2018, 97, No. 121112.

(36) Perdew, J. P.; Burke, K.; Ernzerhof, M. Generalized Gradient Approximation Made Simple. Phys. Rev. Lett. 1996, 77, 3865-3868.

(37) Kweon, K. E.; Hwang, G. S. Hybrid Density Functional Study of the Structural, Bonding, and Electronic Properties of Bismuth Vanadate. Phys. Rev. B 2012, 86, No. 165209.

(38) Yao, S.; Ding, K.; Zhang, Y. The Effects of the Introduction of $\mathrm{Al}$ Atom into Monoclinic $\mathrm{BiVO}_{4}$ : A Theoretical Prediction. Theor. Chem. Acc. 2010, 127, 751-757.

(39) Wang, G.; Ling, Y.; Lu, X.; Qian, F.; Tong, Y.; Zhang, J. Z.; Lordi, V.; Rocha Leao, C.; Li, Y. Computational and Photoelectrochemical Study of Hydrogenated Bismuth Vanadate. J. Phys. Chem. C 2013, 117, 10957-10964.

(40) Cooper, J. K.; Scott, S. B.; Ling, Y.; Yang, J.; Hao, S.; Li, Y.; Toma, F. M.; Stutzmann, M.; Lakshmi, K. V.; Sharp, I. D. Role of Hydrogen in Defining the N-Type Character of $\mathrm{BiVO}_{4}$ Photoanodes. Chem. Mater. 2016, 28, 5761-5771.

(41) Hegner, F. S.; Forrer, D.; Galán-Mascarós, J. R.; López, N.; Selloni, A. Versatile Nature of Oxygen Vacancies in Bismuth Vanadate Bulk and (001) Surface. J. Phys. Chem. Lett. 2019, 10, 6672-6678.

(42) Yin, W.-J.; Wei, S.-H.; Al-Jassim, M. M.; Turner, J.; Yan, Y. Doping Properties of Monoclinic $\mathrm{BiVO}_{4}$ Studied by First-Principles Density-Functional Theory. Phys. Rev. B 2011, 83, No. 155102.

(43) Ding, K.; Chen, B.; Fang, Z.; Zhang, Y.; Chen, Z. Why the Photocatalytic Activity of Mo-Doped $\mathrm{BiVO}_{4}$ Is Enhanced: A Comprehensive Density Functional Study. Phys. Chem. Chem. Phys. 2014, 16, 13465-13476.

(44) Zhao, Z.; Luo, W.; Li, Z.; Zou, Z. Density Functional Theory Study of Doping Effects in Monoclinic Clinobisvanite $\mathrm{BiVO}_{4}$. Phys. Lett. A 2010, 374, 4919-4927.

(45) Zhang, J.; Deng, M.; Ren, F.; Wu, Y.; Wang, Y. Effects of Mo/ $\mathrm{W}$ Codoping on the Visible-Light Photocatalytic Activity of Monoclinic $\mathrm{BiVO}_{4}$ within the GGA + U Framework. RSC Adv. 2016, 6, 12290-12297.

(46) Laraib, I.; Carneiro, M. A.; Janotti, A. Effects of Doping on the Crystal Structure of $\mathrm{BiVO}_{4}$. J. Phys. Chem. C 2019, 123, 2675226757.

(47) Kweon, K. E.; Hwang, G. S.; Kim, J.; Kim, S.; Kim, S. Electron Small Polarons and Their Transport in Bismuth Vanadate: A First Principles Study. Phys. Chem. Chem. Phys. 2015, 17, 256-260.

(48) Rettie, A. J. E.; Chemelewski, W. D.; Emin, D.; Mullins, C. B. Unravelling Small-Polaron Transport in Metal Oxide Photoelectrodes. J. Phys. Chem. Lett. 2016, 7, 471-479.

(49) Wiktor, J.; Ambrosio, F.; Pasquarello, A. Role of Polarons in Water Splitting: The Case of $\mathrm{BiVO}_{4}$. ACS Energy Lett. 2018, 3, 16931697.

(50) Wiktor, J.; Pasquarello, A. Electron and Hole Polarons at the $\mathrm{BiVO}_{4}$-Water Interface. ACS Appl. Mater. Interfaces 2019, 11, $18423-18426$.

(51) Zhang, X.; Huang, Y.; Ma, F.; Zhang, Z.; Wei, X. Influences of Vacancies on the Structural, Electronic and Optical Properties of Monoclinic $\mathrm{BiVO}_{4}$. J. Phys. Chem. Solids 2018, 121, 85-92.

(52) Kühne, T. D.; Iannuzzi, M.; Del Ben, M.; Rybkin, V. V.; Seewald, P.; Stein, F.; Laino, T.; Khaliullin, R. Z.; Schütt, O.; Schiffmann, F.; et al. CP2K: An Electronic Structure and Molecular Dynamics Software Package - Quickstep: Efficient and Accurate Electronic Structure Calculations. J. Chem. Phys. 2020, 152, No. 194103. 
(53) VandeVondele, J.; Hutter, J. Gaussian Basis Sets for Accurate Calculations on Molecular Systems in Gas and Condensed Phases. J. Chem. Phys. 2007, 127, No. 114105.

(54) Goedecker, S.; Teter, M.; Hutter, J. Separable Dual-Space Gaussian Pseudopotentials. Phys. Rev. B 1996, 54, 1703-1710.

(55) Perdew, J. P.; Ernzerhof, M.; Burke, K. Rationale for Mixing Exact Exchange with Density Functional Approximations. J. Chem. Phys. 1996, 105, 9982-9985.

(56) Guidon, M.; Hutter, J.; VandeVondele, J. Robust Periodic Hartree-Fock Exchange for Large-Scale Simulations Using Gaussian Basis Sets. J. Chem. Theory Comput. 2009, 5, 3010-3021.

(57) Guidon, M.; Hutter, J.; VandeVondele, J. Auxiliary Density Matrix Methods for Hartree-Fock Exchange Calculations. J. Chem. Theory Comput. 2010, 6, 2348-2364.

(58) Momma, K.; Izumi, F. VESTA 3 for Three-Dimensional Visualization of Crystal, Volumetric and Morphology Data. J. Appl. Crystallogr. 2011, 44, 1272-1276.

(59) Rossell, M. D.; Agrawal, P.; Borgschulte, A.; Hébert, C.; Passerone, D.; Erni, R. Direct Evidence of Surface Reduction in Monoclinic $\mathrm{BiVO}_{4}$. Chem. Mater. 2015, 27, 3593-3600.

(60) Kim, J. H.; Lee, J. S. Elaborately Modified $\mathrm{BiVO}_{4}$ Photoanodes for Solar Water Splitting. Adv. Mater. 2019, 31, No. 1806938.

(61) Luo, Q.; Zhang, L.; Chen, X.; Tan, O. K.; Leong, K. C. Mechanochemically Synthesized $\mathrm{M}-\mathrm{BiVO}_{4}$ Nanoparticles for Visible Light Photocatalysis. RSC Adv. 2016, 6, 15796-15802.

(62) Appavu, B.; Thiripuranthagan, S.; Ranganathan, S.; Erusappan, E.; Kannan, $\mathrm{K} . \mathrm{BiVO}_{4} / \mathrm{N}$-rGO Nano Composites as Highly Efficient Visible Active Photocatalyst for the Degradation of Dyes and Antibiotics in Eco System. Ecotoxicol. Environ. Saf. 2018, 151, 118126.

(63) Zalfani, M.; van der Schueren, B.; Hu, Z.-Y.; C. Rooke, J.; Bourguiga, R.; Wu, M.; Li, Y.; Tendeloo, G. V.; Su, B.-L. Novel $3 \mathrm{DOM} \mathrm{BiVO}{ }_{4} / \mathrm{TiO}_{2}$ Nanocomposites for Highly Enhanced Photocatalytic Activity. J. Mater. Chem. A 2015, 3, 21244-21256.

(64) Yao, D.; Dong, C.; Bing, Q.; Liu, Y.; Qu, F.; Yang, M.; Liu, B.; Yang, B.; Zhang, H. Oxygen-Defective Ultrathin $\mathrm{BiVO}_{4}$ Nanosheets for Enhanced Gas Sensing. ACS Appl. Mater. Interfaces 2019, 11, 23495-23502.

(65) Wang, S.; Chen, P.; Yun, J.-H.; Hu, Y.; Wang, L. An Electrochemically Treated $\mathrm{BiVO}_{4}$ Photoanode for Efficient Photoelectrochemical Water Splitting. Angew. Chem., Int. Ed. 2017, 56, $8500-8504$ 